\title{
The proof of theorem which characterizes a slant helix
}

\section{Fatih Dogan}

Department of Mathematics, Bartin University, Bartin, Turkey

Received: 16 July 2015, Revised: 24 October 2015, Accepted: 30 October 2015

Published online: 10 April 2016

\begin{abstract}
Firstly, the axis of a slant helix is found. Secondly, the theorem which characterizes a slant helix is proved in detail. The importance of this theorem is stemed from that it has been led to doing many papers about slant helices.
\end{abstract}

Keywords: Slant helix, principal normal indicatrix, the axis, geodesic curvature.

\section{Introduction}

Slant helices are special space curves like Salkowski, Bertrand and Mannheim curves, or general helices. Slant helix concept was first introduced by Izumiya and Takeuchi [1] and then studied by so many authors.

Izumiya and Takeuchi [1] defined slant helices which are generalizations of the notion of general helices. Kula and Yayli [2] investigated the spherical indicatrices of slant helices and showed that the tangent and binormal indicatrices of them are spherical helices. Also, Kula et al. [3] gave some characterizations for slant helices in Euclidean 3-space. Ali and Turgut [4] extended the notion of slant helix from Euclidean 3-space to Euclidean n-space. They introduced type-2 harmonic curvatures of a regular curve and gave necessary and sufficient conditions for a curve to be a slant helix in Euclidean n-space.

Recently, Ali and Turgut [5] researched the position vector of a timelike slant helix in Minkowski 3-space $\mathbb{E}_{1}^{3}$. They determined the parametric representation of the position vector $\psi$ from intrinsic equations in $\mathbb{E}_{1}^{3}$ for the timelike slant helix. Besides, Ali and Lopez [6] looked into slant helices in Minkowski 3-space. They gave characterizations for spacelike, timelike and lightlike slant helices and also investigated tangent indicatrix, binormal indicatrix and involutes of a non-null curve.

More recently, Dogan [7], and Dogan and Yayli [8,9] studied isophote curves on surfaces in Euclidean 3-space and Minkowski 3-space. An isophote curve on a surface can be regarded as a nice consequence of Lambert's cosine law in the optics. Lambert's law states that the intensity of illumination on a diffuse surface is proportional to the cosine of the angle generated between the surface normal vector $N$ and the light vector $d$. According to this law the intensity is irrespective of the actual viewpoint; hence the illumination is the same when viewed from any direction. In other words, isophotes of a surface are curves with the property that their points have the same light intensity from a given source (curves of constant illumination intensity).

When the source light is at infinity, we may consider that the light flow consists of parallel lines. They $[7,8,9]$ showed that the there is a close relation between isophote curves and slant helices, i.e., a curve which is both a geodesic and a 
slant helix is an isophote curve.

This paper is organized as follows. Section 2 presents basic concepts concerning curve and surface theory in $\mathbb{E}^{3}$. Firstly, the axis of a slant helix is found and secondly, the theorem which characterizes a slant helix is proved in section 3.

\section{Preliminaries}

In this section, we give some basic notions about curves and surfaces. The differential geometry of curves starts with a smooth map of $s$; let us call it $\alpha: I \subset \mathbb{R} \longrightarrow \mathbb{E}^{3}$ that parametrizes a spatial curve and it will be denoted again with $\alpha$. The curve $\alpha$ is parametrized by arc-length if $\left\|\alpha^{\prime}(s)\right\|=1$ (unit-speed), where $\alpha^{\prime}(s)$ is the first derivative of $\alpha$ with respect to $s$. Let $\alpha: I \subset \mathbb{R} \longrightarrow \mathbb{E}^{3}$ be a regular curve with an arc-length parameter $s$ and $\kappa(s)=\left\|\alpha^{\prime \prime}(s)\right\|$, where $\kappa$ is the curvature of $\alpha$, and $\alpha^{\prime \prime}$ is the second derivative of $\alpha$. For $\kappa>0$, the Frenet frame $\{T, N, B\}$ is well-defined along the curve $\alpha$ and as follows.

$$
\begin{gathered}
T(s)=\alpha^{\prime}(s), \\
N(s)=\frac{\alpha^{\prime \prime}(s)}{\left\|\alpha^{\prime \prime}(s)\right\|}, \\
B(s)=T(s) \times N(s),
\end{gathered}
$$

where $T, N$ and $B$ are the tangent, the principal normal, and the binormal of $\alpha$, respectively. The derivative of the Frenet frame (Frenet equations) are given by

$$
\begin{gathered}
T^{\prime}(s)=\kappa(s) N(s), \\
N^{\prime}(s)=-\kappa(s) T(s)+\tau(s) B(s), \\
B^{\prime}(s)=-\tau(s) N(s),
\end{gathered}
$$

where $\tau(s)=\frac{\left\langle\alpha^{\prime}(s) \times \alpha^{\prime \prime}(s), \alpha^{\prime \prime \prime}(s)\right\rangle}{\kappa^{2}(s)}$ is the torsion of $\alpha ; "\langle$,$\rangle " is the standart inner product, and " \times$ " is the cross product on $\mathbb{R}^{3}$.

Let $\mathbb{M}$ be a regular surface and $\alpha: I \subset \mathbb{R} \longrightarrow \mathbb{M}$ be a unit-speed curve. Then the Darboux frame $\{T, Y=U \times T, U\}$ is well-defined along the curve $\alpha$, where $T$ is the tangent of $\alpha$ and $U$ is the unit normal of $\mathbb{M}$. If we rotate the Darboux frame $\{T, Y=U \times T, U\}$ by $\phi$ about $T$, we obtain the Frenet frame $\{T, N, B\}$.

$$
\begin{gathered}
{\left[\begin{array}{l}
T \\
N \\
B
\end{array}\right]=\left[\begin{array}{ccc}
1 & 0 & 0 \\
0 & \cos \phi & \sin \phi \\
0 & -\sin \phi & \cos \phi
\end{array}\right]\left[\begin{array}{l}
T \\
Y \\
U
\end{array}\right]} \\
T=T \\
N=\cos \phi Y+\sin \phi U \\
B=-\sin \phi Y+\cos \phi U
\end{gathered}
$$


The derivative formulas for the Darboux frame are given by

$$
\begin{gathered}
T^{\prime}=k_{g} Y+k_{n} U, \\
Y^{\prime}=-k_{g} T+\tau_{g} U, \\
U^{\prime}=-k_{n} T-\tau_{g} Y,
\end{gathered}
$$

where "' " denotes the derivative of $T, B$, and $N$ with respect to $s$ along the curve $U 3 b 1 ; k_{n}, k_{g}$, and $\tau_{g}$ are the normal curvature, the geodesic curvature, and the geodesic torsion of $\alpha$, respectively. With the above notations, let $\phi$ denote the angle between the surface normal $U$ and the binormal $B$. Using equations (2), (3) and (4) we obtain

$$
\begin{aligned}
\kappa^{2} & =k_{g}^{2}+k_{n}^{2}, \\
k_{g} & =\kappa \cos \phi, \\
k_{n} & =\kappa \sin \phi, \\
\tau_{g} & =\tau-\phi^{\prime} .
\end{aligned}
$$

Let $\alpha: I \subset \mathbb{R} \longrightarrow \mathbb{E}^{3}$ be a regular curve with an arc-length parameter $s$ and $\kappa>0$. Since $\|N\|=1$, the curve $\beta: I \subset \mathbb{R} \longrightarrow \mathbb{S}^{2}$, $\beta(s)=N(s)$ lies on the unit sphere $\mathbb{S}^{2}$. It is called the principal normal indicatrix of $\alpha$.

\section{A Slant helix and its axis}

In this section, we find the fixed vector (axis) of a slant helix. By means of this axis, we prove the theorem which characterizes a slant helix in detail. A space curve whose its principal normal vectors make a constant angle with a fixed vector is called a slant helix. Let $\alpha: I \subset \mathbb{R} \longrightarrow \mathbb{E}^{3}$ be a unit-speed slant helix with $\kappa(s) \neq 0$. By the definition of slant helix

$$
\langle N, d\rangle=\cos \theta,
$$

where $N$ is the principal normal, $d$ is the fixed vector of $\alpha$, and $\theta$ is the constant angle between $N$ and $d$, respectively. If we differentiate Eq.(6) with respect to $s$ along the curve $\alpha$ and then use the Frenet equations, we obtain

$$
\begin{gathered}
\left\langle N^{\prime}, d\right\rangle=0 \\
\langle-\kappa T+\tau B, d\rangle=0 \\
\kappa\langle T, d\rangle=\tau\langle B, d\rangle \\
\langle T, d\rangle=\frac{\tau}{\kappa}\langle B, d\rangle .
\end{gathered}
$$

If we take $\langle B, d\rangle=c$, we get

$$
d=\frac{\tau}{\kappa} c T+\cos \theta N+c B
$$

Since $\|d\|=1$, it follows that

$$
\begin{aligned}
& \frac{\tau^{2}}{\kappa^{2}} c^{2}+\cos ^{2} \theta+c^{2}=1 \\
& \left(\frac{\tau^{2}}{\kappa^{2}}+1\right) c^{2}=\sin ^{2} \theta \\
& c=\mp \frac{\kappa}{\sqrt{\kappa^{2}+\tau^{2}}} \sin \theta .
\end{aligned}
$$


Therefore, the vector $d$ can be written as

$$
d=\mp \frac{\tau}{\sqrt{\kappa^{2}+\tau^{2}}} \sin \theta T+\cos \theta N \mp \frac{\kappa}{\sqrt{\kappa^{2}+\tau^{2}}} \sin \theta B
$$

Here, $d$ is actually a constant vector. By differentiating Eq.(7) with respect to $s$ along the curve $\alpha$, we obtain

$$
\begin{gathered}
\left\langle N^{\prime \prime}, d\right\rangle=0 \\
\left\langle\kappa^{\prime} T+\left(\kappa^{2}+\tau^{2}\right) N-\tau^{\prime} B, \mp \frac{\tau}{\sqrt{\kappa^{2}+\tau^{2}}} \sin \theta T+\cos \theta N \mp \frac{\kappa}{\sqrt{\kappa^{2}+\tau^{2}}} \sin \theta B\right\rangle=0 \\
\mp \frac{\kappa^{\prime} \tau-\tau^{\prime} \kappa}{\left(\kappa^{2}+\tau^{2}\right)^{3 / 2}} \tan \theta+1=0 \\
\tan \theta=\mp \frac{\left(\kappa^{2}+\tau^{2}\right)^{3 / 2}}{\tau^{\prime} \kappa-\kappa^{\prime} \tau} \\
\cot \theta=\mp \frac{\kappa^{2}}{\left(\kappa^{2}+\tau^{2}\right)^{3 / 2}}\left(\frac{\tau}{\kappa}\right)^{\prime}
\end{gathered}
$$

From Eq.(8) and the Frenet equations, the derivative of $d$ becomes

$$
\begin{aligned}
d^{\prime} & =\mp\left[\sin \theta\left(\frac{\tau}{\sqrt{\kappa^{2}+\tau^{2}}}\right)^{\prime} T+\sin \theta \frac{\tau}{\sqrt{\kappa^{2}+\tau^{2}}}(\kappa N)\right]+\cos \theta(-\kappa T+\tau B) \\
& \mp\left[\sin \theta\left(\frac{\kappa}{\sqrt{\kappa^{2}+\tau^{2}}}\right)^{\prime} B+\sin \theta \frac{\kappa}{\sqrt{\kappa^{2}+\tau^{2}}}(-\tau N)\right] \\
& =\left[-\kappa \mp \tan \theta\left(\frac{\tau}{\sqrt{\kappa^{2}+\tau^{2}}}\right)^{\prime}\right] T+\left[\tau \mp \tan \theta\left(\frac{\kappa}{\sqrt{\kappa^{2}+\tau^{2}}}\right)^{\prime}\right] B .
\end{aligned}
$$

If we substitute Eq.(9) in the last equality above, we get

$$
\begin{aligned}
d^{\prime} & =\left[-\kappa+\frac{\left(\kappa^{2}+\tau^{2}\right)^{3 / 2}}{\tau^{\prime} \kappa-\kappa^{\prime} \tau}\left(\frac{\tau^{\prime}\left(\kappa^{2}+\tau^{2}\right)-\tau\left(\kappa \kappa^{\prime}+\tau \tau^{\prime}\right)}{\left(\kappa^{2}+\tau^{2}\right)^{3 / 2}}\right)\right] T \\
& +\left[\tau+\frac{\left(\kappa^{2}+\tau^{2}\right)^{3 / 2}}{\tau^{\prime} \kappa-\kappa^{\prime} \tau}\left(\frac{\kappa^{\prime}\left(\kappa^{2}+\tau^{2}\right)-\kappa\left(\kappa \kappa^{\prime}+\tau \tau^{\prime}\right)}{\left(\kappa^{2}+\tau^{2}\right)^{3 / 2}}\right)\right] B
\end{aligned}
$$

By a direct calculation, it can be seen that the coefficients of $T$ and $B$ are zero. Then $d^{\prime}=0$, in other words, $d$ is a constant vector.

Theorem 1. A unit-speed curve $\alpha: I \subset \mathbb{R} \longrightarrow \mathbb{E}^{3}$ with $\kappa(s) \neq 0$ is a slant helix if and only if

$$
\sigma(s)=\mp\left(\frac{\kappa^{2}}{\left(\kappa^{2}+\tau^{2}\right)^{3 / 2}}\left(\frac{\tau}{\kappa}\right)^{\prime}\right)(s)
$$

is a constant function [1].

Proof. The vectors that make a constant angle with a fixed vector construct a cone. Then the unit vectors in $\mathbb{E}^{3}$, which make a constant angle with a fixed vector construct a circular cone whose the base curve lies on the unit sphere $\mathbb{S}^{2}$. Therefore, $\alpha$ is the unit-speed slant helix if and only if its principal normal indicatrix is a circle on the unit sphere $\mathbb{S}^{2}$. In other words, if we compute the normal indicatrix $\beta: I \subset \mathbb{R} \longrightarrow \mathbb{S}^{2}, \beta(s)=N(s)$ along the curve $\alpha$, the geodesic curvature of $\beta$ becomes 
$\sigma(s)$ as obtained below.

$$
\begin{aligned}
N^{\prime} & =-\kappa T+\tau B \\
N^{\prime \prime} & =-\kappa^{\prime} T-\left(\kappa^{2}+\tau^{2}\right) N+\tau^{\prime} B, \\
N^{\prime} \times N^{\prime \prime} & =\tau\left(\kappa^{2}+\tau^{2}\right) T+\kappa^{2}\left(\frac{\tau}{\kappa}\right)^{\prime} N+\kappa\left(\kappa^{2}+\tau^{2}\right) B, \\
\kappa_{\beta} & =\frac{\left\|N^{\prime} \times N^{\prime \prime}\right\|}{\left\|N^{\prime}\right\|^{3}}, \\
& =\sqrt{\frac{\left(\kappa^{2}+\tau^{2}\right)^{3}+\left(\kappa^{2}\left(\frac{\tau}{\kappa}\right)^{\prime}\right)^{2}}{\left(\kappa^{2}+\tau^{2}\right)^{3}}},
\end{aligned}
$$

where $\kappa_{\beta}$ is the curvature of $\beta$. Let $k_{g}$ and $k_{n}$ be the geodesic curvature and normal curvature of $\beta$ on $\mathbb{S}^{2}$, respectively. Since the normal curvature $k_{n}=1$ on $\mathbb{S}^{2}$, if we substitute $k_{n}$ and $\kappa_{\beta}$ in the following equation, we get the geodesic curvature $k_{g}$ as follows.

$$
\begin{gathered}
k_{g}^{2}+k_{n}^{2}=\left(\kappa_{\beta}\right)^{2}, \\
k_{g}(s)=\sigma(s)=\cot \theta=\mp\left(\frac{\kappa^{2}}{\left(\kappa^{2}+\tau^{2}\right)^{3 / 2}}\left(\frac{\tau}{\kappa}\right)^{\prime}\right)(s),
\end{gathered}
$$

where $\theta$ is the constant angle between the principal normal $N$ and the axis $d$. Because of the fact that $\theta$ is a constant, $\sigma(s)$ is a constant. Then, a unit-speed curve $\alpha: I \subset \mathbb{R} \longrightarrow \mathbb{E}^{3}$ is a slant helix if and only if the spherical image (indicatrix) of its principal normal $\beta: I \subset \mathbb{R} \longrightarrow \mathbb{S}^{2}$ is a circle.

\section{References}

[1] S. Izumiya and N. Takeuchi, New special curves and developable surfaces, Turk. J. Math. 28, 153-163, 2004.

[2] L. Kula and Y. Yayli , On slant helix and its spherical indicatrix, Applied Mathematics and Computation 169 (1), 600-607, 2005.

[3] L. Kula, N. Ekmekci, Y. Yayli , K. Ilarslan, Characterizations of slant helices in Euclidean 3-space, Turk. J. Math. 34, 261-273, 2010.

[4] A.T. Ali and M. Turgut, Some characterizations of slant helices in the Euclidean space $\mathbb{E}^{n}$, Hacettepe Journal of Mathematics and Statistics 39 (3), 327-336, 2010.

[5] A.T. Ali and M. Turgut, Position vector of a time-like slant helix in Minkowski 3-space, J. Math. Anal. Appl. 365, 559-569, 2010.

[6] A.T. Ali and R. Lopez, Slant helices in Minkowski space $\mathbb{E}_{1}^{3}$, J. Korean Math. Soc. 48 (1), 159-167, 2011.

[7] F. Dogan, Isophote curves on timelike surfaces in Minkowski 3-Space, An. Stiint. Univ. Al. I. Cuza Iasi. Mat. (S.N.), DOI:10.2478/aicu-2014-0020.

[8] F. Dogan and Y.Yayli , Isophote curves on spacelike surfaces in Lorentz-Minkowski space $\mathbb{E}_{1}^{3}$. arXiv:1203.4388.

[9] F. Dogan and Y.Yayli , On isophote curves and their characterizations, Turk. J. Math., 39 (5), 650-664, 2015. 